\title{
Cryptogenic cervical intramedullary abscess with rapidly progressive myelopathy: illustrative case
}

\author{
Nebras M. Warsi, MD, CM, ${ }^{1}$ Ann Wilson, LLB, ${ }^{1}$ Armaan K. Malhotra, MD, ${ }^{1}$ Jerry C. Ku, MD, ${ }^{1}$ Ahmed A. Najjar, MBBS, MSc, FRCSC, ${ }^{2}$ \\ Esther Bui, MD, FRCP(C), ${ }^{3}$ Michael Baker, MD, FRCP(C), ${ }^{3}$ Eric Bartlett, MD, FRCP(C), ${ }^{4}$ and Mojgan Hodaie, MD, MSc, FRCS(C) ${ }^{1,2}$ \\ ${ }^{1}$ Division of Neurosurgery, Department of Surgery, University of Toronto, Toronto, Ontario, Canada; 'Division of Neurosurgery, Toronto Western Hospital, \\ University Health Network, Toronto, Ontario, Canada; ${ }^{3}$ Department of Medicine, Toronto General Hospital, University Health Network, Toronto, Ontario, Canada; and ${ }^{4}$ Division \\ of Neuroradiology, Department of Medical Imaging, Toronto Western Hospital, Toronto, Ontario, Canada
}

BACKGROUND The purpose of the present case report is to highlight the presentation, workup, clinical decision making, and operative intervention for a 68-year-old woman who developed rapidly progressive myelopathy secondary to idiopathic cervical intramedullary abscess.

OBSERVATIONS The patient underwent laminectomy and aspiration/biopsy of the lesion. Intraoperatively, division of the posterior median sulcus released a large volume of purulent material growing the oral pathogens Eikenella corrodens and Gemella morbillorum. Broad-spectrum antibiotics were initiated postoperatively. At the 6-month follow-up, the patient had almost completely recovered with some persistent hand dysesthesia. Complete infectious workup, including full dental assessment and an echocardiogram, failed to reveal the source of her infection.

LESSONS The authors report the first case of cryptogenic spinal intramedullary abscess secondary to Eikenella spp. and Gemella spp. coinfection. Intramedullary abscesses are exceptionally rare and most commonly develop in children with dermal sinus malformations or in the context of immunosuppression. In adults without risk factors, they can readily be mistaken for more common pathologies in this age group, such as intramedullary neoplasms or demyelinating disease. Prompt diagnosis and management based on rapidly progressive myelopathy, assessment of infectious risk factors and/or symptoms, and targeted imaging are critical to avoid potentially devastating neurological sequelae.

https://thejns.org/doi/abs/10.3171/CASE2035

KEYWORDS case report; spinal abscess; intramedullary abscess; CNS infection; neuroinfectious disease

Intramedullary spinal cord abscess (ISCA) is a rare but potentially devastating clinical entity associated with significant neurological morbidity and death. ${ }^{1}$ Since its initial characterization by Hart in 1830 , there have been approximately 100 cases described in the literature. ${ }^{2,3}$ Unlike intracranial abscesses, these infections rarely arise without a direct source of infection, such as in children with dermal sinus malformations, extension from vertebral osteomyelitis, postsurgical infections, or recurrent bacterial meningitis. ${ }^{4-8}$ When no such source is found, the infection is termed "cryptogenic" and most commonly arises due to an underlying risk factor for abscess development such as immunosuppression, intravenous (IV) drug use history, poorly controlled diabetes mellitus, or pulmonary arteriovenous malformations. ${ }^{6-8}$
In adult patients without such risk factors, cryptogenic ISCA is particularly rare and can therefore easily be overlooked or mistaken for more common diagnoses such as spinal intramedullary neoplasia, demyelinating disease, resolving hematoma, or infarction. ${ }^{9}$

\section{Illustrative Case}

A 68-year-old woman presented with acute-onset progressive neck pain, arm paresthesia, and gait disturbance over the preceding week. Right-sided shoulder pain was first noticed after playing baseball, which was initially thought to be due to muscular strain. However, over the next few days, this was followed by bilateral numbness and paresthesia to the C8-T1 dermatomes. At the time of presentation, the patient had also developed gait imbalance and numbness in the

ABBREVIATIONS ADC = apparent diffusion coefficient; $C T$ = computed tomography; DWI = diffusion-weighted imaging; ISCA = intramedullary spinal cord abscess; IV = intravenous; $M R I=$ magnetic resonance imaging.

INCLUDE WHEN CITING Published January 25, 2021; DOI: 10.3171/CASE2035.

SUBMITTED September 25, 2020. ACCEPTED September 29, 2020.

(c) 2021 The authors, CC BY-NC-ND 4.0 (http://creativecommons.org/licenses/by-nc-nd/4.0/). 

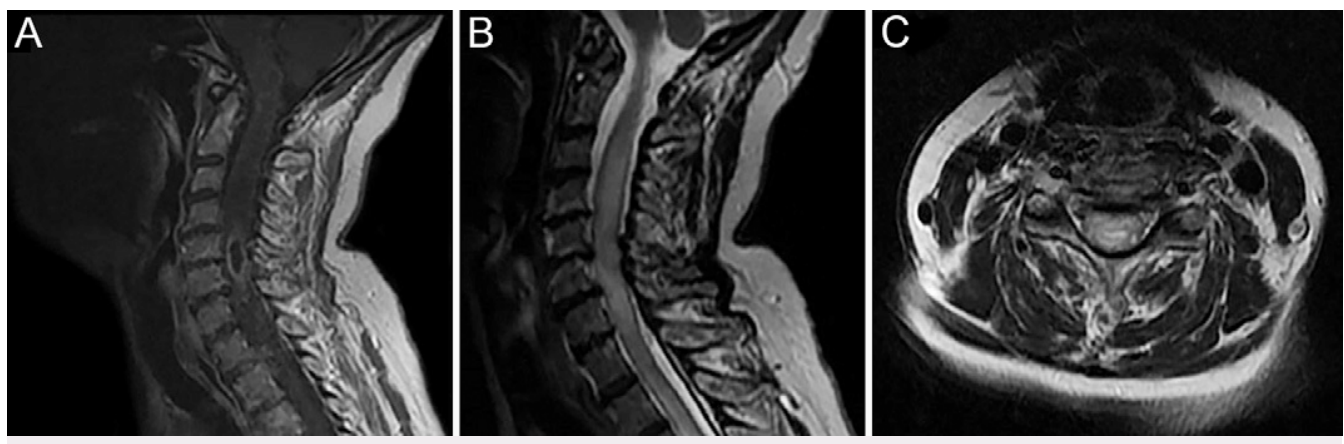

FIG. 1. Initial spinal imaging. A: Sagittal T1-weighted MRI scan with IV contrast demonstrating a cervical intramedullary lesion with a T1-hypointense core and surrounding ring enhancement. Sagittal (B) and axial (C) T2weighted MRI scans demonstrate significant perilesional edema spanning multiple spinal levels.

bilateral S1 dermatomes. She had a sensation of a full bladder but no reported urinary or fecal incontinence. Her past medical history was significant for mitral valve prolapse and breast cancer with prior mastectomy in 2006 and successfully treated with tamoxifen from 2006 to 2012. Past medical history was negative for risk factors of infection such as cardiac or pulmonary malformations, diabetes mellitus, recent dental intervention, or IV drug use.

On examination, the patient's vital signs were normal, and she was afebrile. Her laboratory results were unremarkable, and the results of her blood cultures were negative. Initial testing revealed $4 / 5$ power in the right first dorsal interosseous, full power in the remaining myotomes, and a wide-based, ataxic gait. However, over the course of assessment in the emergency department, her strength rapidly deteriorated to $4 / 5$ power bilaterally in the arms, and she became unable to ambulate and had significantly diminished sensation in a "cape-like" distribution along the C2-T1 dermatomes. Her pinprick and fine-touch sensation were also diminished in the bilateral lower extremities. She had diffuse bilateral hyperreflexia with normal tone throughout. No Hoffman's reflex was present, and her plantar response was equivocal bilaterally. Her postvoid residual was significantly elevated at $>500 \mathrm{~mL}$.

Our impressions at this stage were of a progressive myelopathy, and therefore magnetic resonance imaging (MRI) of the whole spine with and without gadolinium was ordered. This revealed a C5/6 ringenhancing intramedullary lesion with a T1-hypointense central core (Fig. 1A). T2 hyperintensity with significant associated edema spanned from C2-T3 (Fig. 1B and C). There was no evidence of associated syringomyelia, vertebral osteomyelitis, or occult spinal dysraphism. Imaging of the remainder of the neuraxis did not identify other lesions. The predominant opinion was that this lesion most likely represented either a primary intramedullary tumor such as an astrocytomal ependymoma or an intramedullary metastasis, given the patient's known cancer history. Given that no other lesions were seen in the neuraxis and that no primary could be identified on a computed tomography (CT) scan of the thorax, abdomen, and pelvis, the possibility of an infectious etiology was considered. Urgent follow-up MRI with diffusion-weighted imaging (DWI)/apparent diffusion coefficient (ADC) sequences was therefore arranged. Unlike in cranial imaging, because of technical limitations, DWI is not part of standard spinal imaging protocols, and it was performed on the basis of specific clinical suspicion for intramedullary infection. ${ }^{9}$ This revealed (Fig. 2) diffusion restriction of the lesion, supporting the diagnosis of a spinal intramedullary abscess.
In light of the patient's progressive deterioration, informed consent was obtained for urgent laminectomy and aspiration/biopsy of the lesion with the goals of surgical decompression and tissue diagnosis. Intraoperatively, division of the posterior median sulcus led to the immediate egress of purulent material, strongly supporting the diagnosis of ISCA. Culture of this fluid showed growth of the oral pathogens Eikenella corrodens and Gemella morbillorum. Postoperatively, the patient was started on broad-spectrum antibiotics, which were then narrowed to ongoing ceftriaxone and metronidazole treatment. On the basis of the relative rarity of these pathogens, she underwent further investigations for assessment of possible underlying sources of infection. This included dental assessment, dental radiographs, a transesophageal echocardiogram, and a colonoscopy. These did not reveal any underlying source of infection, confirming cryptogenic ISCA.

Immediately following surgery, the patient improved neurologically. By 1 week postoperatively, she had regained signification function, with residual difficulties in fine finger movements and gait imbalance, and she was transferred to inpatient rehabilitation with an approximately 4-month course of antibiotics. Ather 6-month follow-up visit, the patient
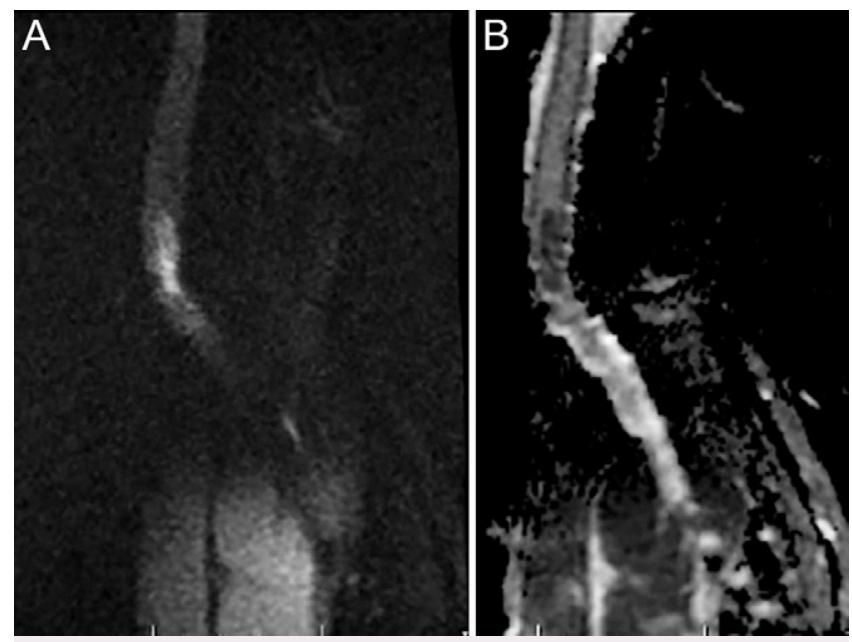

FIG. 2. A: DWI-based MRI scan of the lesion. B: Corresponding ADC map confirming high diffusion signal, supporting the diagnosis of ISCA. 
was found to have almost full recovery of her neurological function. Her predominant residual neurological deficit relates to dysesthesia in both hands. Repeat MRI at 6 months demonstrated complete resolution of the previously seen ISCA.

\section{Discussion \\ Observations}

ISCA is an exceptionally rare pathology, particularly in adults. Of the 197 reported cases since 1830, 54\% were in children younger than 12 years of age; $71 \%$ of patients were male; and $44 \%$ of patients presented with acute symptom onset over a 1 -week period. ${ }^{10}$ This also contrasts with the much higher prevalence of intracranial abscesses. Previous groups have postulated a structural explanation, suggesting that the lack of dural sinuses, smaller subarachnoid space, and centripetal versus centrifugal blood flow differences in the spine and brain, respectively, lead to less abscess formation in the spinal cord; a similar phenomenon has been described for the relative rarity of intramedullary metastases as compared with intracranial metastases..$^{5,11,12}$ Risk factors associated with ISCA include spinal dysraphism, intradural neoplasia, bacterial meningitis, bacteremia, and postoperative infections. ${ }^{4,6-8}$ Furthermore, spinal degenerative changes have been postulated to play a role in seeding transient bacteremia through chronic inflammation and microtrauma. ${ }^{5}$

With regard to cryptogenic ISCA, some evidence suggests that these infections are more common in the cervical and upper thoracic regions because of the larger numbers and caliber of blood vessels supplying these areas, allowing greater accessibility for infection. ${ }^{6,13}$ This cervicothoracic predilection has also been described with intradural spinal cord metastases. ${ }^{14,15}$ Compiling results from 2 reviews, there were 40 cases of cryptogenic ISCA from 1830 to 2007; however, an underlying risk factor, such as poorly controlled diabetes mellitus, IV drug use, or pulmonary arteriovenous malformation, was identifiable in 35 of these cases. ${ }^{1,6}$ Dating back to 1830 , only 5 cases were found in which a cryptogenic ISCA presented in the absence of any risk factor for such an infection.

Our patient presented with cryptogenic ISCA secondary to $E$. corrodens and G. morbillorum. E. corrodens is a fastidious, gramnegative bacillus that has been described in up to $20 \%$ of gramnegative bacilli isolates from intracerebral abscess cases attributable to oral sources. ${ }^{16,17}$ G. morbillorum is a facultative, anaerobic, grampositive coccus and has been described in 11 total intracranial cases in the literature. ${ }^{18,19}$ Because these are both oral pathogens, the treating infectious disease team postulated that possible mechanisms of inoculation would be direct spread from the oropharynx to the retropharyngeal space and eventually the cervical spine or transient bacteremia with seeding in the intradural intramedullary space. However, a CT scan failed to demonstrate any evidence of retropharyngeal infection, and the patient's history was negative for endocarditis, retropharyngeal abscess, or odynophagia. The identification of Gemella spp. and Eikenella spp. microbes in this case report therefore represents the first described spinal cord abscess from either of these organisms and the first instance of their central nervous system coinfection.

MRI with DWI proved to be instrumental in our diagnostic workup because up to $60 \%$ of ISCA cases can present without systemic signs of infection. ${ }^{6}$ In conjunction with a rapid clinical decline, the pattern of diffusion restriction in this case strongly supported an infectious process (Fig. 2). 2,5,8,14,15,20,21 Neoplastic lesion cores, unlike ISCA, tend to display no signal on DWI or DWI hypointensity and high ADC signal intensity signifying necrosis, though diffusion restriction can rarely be seen in the case of an associated hematoma. ${ }^{9}$ In this case, the degree of cord edema and the restricting lesion core provided sufficient evidence to proceed with surgical intervention with the goals of decompression, tissue diagnosis, and culture of the aspirate fluid. This confirmed the diagnosis of polymicrobial ISCA. Though the use of DWI in spinal imaging is not commonplace, in our patient's clinical context, this targeted imaging greatly informed management and led to a favorable outcome.

Prompt initiation of antimicrobial therapy in treating ISCA is critical. Chan and Gold conducted a retrospective review of 25 ISCA cases from 1977 to 1997 and compared outcomes with those in 42 reported cases before the antibiotic era from 1830 to $1944 .{ }^{6}$ In the modern group, 21 patients underwent surgery and antibiotic therapy, 2 patients had myelotomy alone, and 2 were managed with antibiotics alone. Of the 2 fatal outcomes, 1 was in the surgery-only group and the other was in the combined group. Both were in patients with concomitant cerebral abscesses. Of the 23 patients who survived, 7 had complete resolution of neurological deficits, and all were in the combined surgical/antibiotic treatment group. The frequency of surgical intervention in the preantibiotic group was not detailed. Overall mortality was $8 \%$ in the modern group compared with $90 \%$ in the preantibiotic group, suggesting that prompt management with appropriate antimicrobials must be initiated in cases of ISCA.

The role of surgery in treating ISCA is less clear. Kurita et al. compared combined surgical/antimicrobial management with antibiotics alone. ${ }^{1}$ They performed a review of the literature from 1998 to 2007 , identifying 20 patients who underwent combined treatment and 6 who were treated with antibiotics alone. Interestingly, they found similar neurological outcomes documented at the long-term follow-up for both groups. There was a statistically nonsignificant effect of a poorer neurological outcome in conservatively managed patients treated with less than 6 weeks of antibiotics, as well as for delayed initiation of antibiotic therapy. Similar to Chan and Gold's findings, the single death occurred in a patient with concomitant disseminated nocardiosis and cranial abscesses. ${ }^{6,22}$ Unfortunately, Kurita's work is the only report we have found comparing combined surgical/antimicrobial treatment with antibiotic therapy alone, and the group for comparison is quite small. Given this limitation, we suggest that although antibiotic treatment at a minimum should be required in all cases of ISCA, surgery may be beneficial in cases with significant cord compression and rapid symptom progression, accessible lesions, associated anatomical abnormalities requiring correction, or in cases in which tissue diagnosis is required to exclude a neoplastic or other lesion. In our case, prompt surgical management allowed confirmation of diagnosis, decompression of an edematous cord, and collection of cultures/sensitivities to guide antimicrobial therapy in the absence of previously isolated organisms.

\section{Lessons}

In our patient's clinical context, there was very little to argue for a diagnosis of spinal infection. On the basis of her age, previous cancer history, and absence of risk factors, the initial impression was that her symptoms and imaging findings reflected intramedullary neoplasia. Indeed, even referral for urgent radiotherapy had been considered. However, a rapidly progressive clinical course and significant perilesional edema observed using MRI did not fit with a neoplastic process, and we therefore considered the unlikely differential of ISCA, later confirmed with targeted spinal DWI/ADC sequences and subsequent abscess aspiration. Neurosurgical trainees and practitioners should be aware of this rare and pernicious clinical entity, which may present as 
rapidly progressive myelopathy and an intramedullary lesion, without systemic signs, symptoms, or risk factors of infection. Prompt recognition with DWI and early surgical intervention are critical to avoid possibly severe neurological sequelae.

\section{References}

1. Kurita N, Sakurai Y, Taniguchi M, et al. Intramedullary spinal cord abscess treated with antibiotic therapy-case report and review. Neurol Med Chir (Tokyo). 2009;49(6):262-268.

2. Sinha P, Parekh T, Pal D. Intramedullary abscess of the upper cervical spinal cord. Unusual presentation and dilemmas of management: case report. Clin Neurol Neurosurg. 2013;115(9):1845-1850.

3. Hart J. Case of encysted abscess in the center of the spinal cord. Dublin Hosp Rep. 1830;5:522-524.

4. Barbarawi MDA, Khriesat W, Qudsieh S, et al. Management of intramedullary spinal cord abscess: experience with four cases, pathophysiology and outcomes. Eur Spine J. 2009;18(5): 710-717.

5. Kulkarni A, Chu G, Fehlings MG. Pyogenic intradural abscess: a case report. Spine (Phila Pa 1976). 2007;32(12):E354-E357.

6. Chan CT, Gold W. Intramedullary abscess in the spinal cord in the antibiotic era: clinical features, microbial etiologies, trends in pathogenesis, and outcomes. Clin Infect Dis. 1998;27(3):619-626.

7. Takebe $\mathrm{N}$, Iwasaki K, Hashikata $\mathrm{H}$, et al. Intramedullary spinal cord abscess and subsequent granuloma formation: a rare complication of vertebral osteomyelitis detected by diffusionweighted magnetic resonance imaging. Neurosurg Focus. 2014; 37(2):E12.

8. Kim MS, Ju Cl, Kim SW, et al. Recurrent bacterial meningitis accompanied by a spinal intramedullary abscess. J Korean Neurosurg Soc. 2012;51(6):380-382.

9. Dörflinger-Hejlek E, Kirsch EC, Reiter $\mathrm{H}$, et al. AJNR Am J Neuroradiol. 2010;31(9):1651-1652.

10. Lapointe S, Legault C, Altman R, et al. Intramedullary spinal cord abscess; descriptive review of the literature [abstract]. Neurology. 2016;86(16 Suppl):2.015.

11. Thome C, Krauss JK, Zevgaridis D, et al. Pyogenic abscess of the filum terminale: case report. J Neurosurg Spine. 2001;95(1):100-104.

12. Hammack JE. Spinal cord disease in patients with cancer. Continuum (Minneap Minn). 2012;18(2):312-327.

13. Gillilan LA. The arterial blood supply of the human spinal cord. J Comp Neurol. 1958;110(1):75-103.

14. Kalayci M, Cağavi $F$, Gül $S$, et al. Intramedullary spinal cord metastases: diagnosis and treatment-an illustrated review. Acta Neurochir (Wien). 2004;146:1347-1354.
15. Payer S, Mende KC, Westphal M, et al. Intramedullary spinal cord metastasis: an increasingly common diagnosis. Neurosurg Focus. 2017;39(2):E15.

16. Moazzam AA, Rajagopal SM, Sedghizadeh PP, et al. Intracranial bacterial infections of oral origin. J Clin Neurosci. 2015;22(5): 800-806.

17. Suwanagool S, Rothkopf MM, Smith SM, et al. Pathogenicity of Eikenella corrodens in humans. Arch Intern Med. 1983;143(12): 2265-2268.

18. Abu-Heija AA, Ajam M, Veltman J. Gemella morbillorum cryptogenic brain abscess: a case report and literature review. Cureus. 2018;10(11):e3612.

19. Benedetti P, Rassu M, Branscombe M, et al. Gemella morbillorum: an underestimated aetiology of central nervous system infection? J Med Microbiol. 2009;58(12):1652-1656.

20. Bakhsheshian J, Kim PE, Attenello FJ. Intramedullary cervical spinal cord abscess. World Neurosurg. 2017;106: 1049.e1-1049.e2.

21. Kim YJ, Chang KH, Song IC, et al. Brain abscess and necrotic brain tumor: discrimination with signal intensity on diffusionweighted MR imaging. AJR Am J Roentgenol. 1998;171(6): 1487-1490.

22. Durmaz R, Atasoy MA, Durmaz G, et al. Multiple nocardial abscesses of cerebrum, cerebellum and spinal cord, causing quadriplegia. Clin Neurol Neurosurg. 2001;103(1):59-62.

\section{Disclosures}

The authors report no conflict of interest concerning the materials or methods used in this study or the findings specified in this paper.

\section{Author Contributions}

Conception and design: Hodaie, Warsi, Ku, Bui. Acquisition of data: Hodaie, Warsi, Ku, Najjar, Bui, Baker, Bartlett. Analysis and interpretation of data: Hodaie, Warsi, Malhotra, Ku, Bui, Baker, Bartlett. Drafting the article: Warsi, Malhotra, Najjar. Critically revising the article: Hodaie, Warsi, Malhotra, Ku, Baker. Reviewed submitted version of manuscript: Hodaie, Warsi, Wilson, Malhotra, Ku, Bui, Bartlett. Approved the final version of the manuscript on behalf of all authors: Hodaie. Administrative/technical/material support: Warsi. Study supervision: Bartlett.

\section{Correspondence}

Mojgan Hodaie: Toronto Western Hospital, University Health Network, Toronto, ON, Canada. mojgan.hodaie@uhn.ca. 